\title{
Multiscale lensless imaging with a plasma x-ray laser
}

\section{Davide Bleiner, Mabel Ruiz-Lopez}

Davide Bleiner, Mabel Ruiz-Lopez, "Multiscale lensless imaging with a plasma x-ray laser," Proc. SPIE 11886, International Conference on X-Ray Lasers 2020, 118860P (8 July 2021); doi: 10.1117/12.2596041

SPIE. Event: XVII International Conference on X-Ray Lasers, 2020, Online Only 


\title{
Multiscale Lensless Imaging with a Plasma X-ray Laser
}

\author{
Davide Bleiner and Mabel Ruiz-Lopez \\ ${ }^{a}$ Swiss Federal Laboratories for Materials \& Technology (Empa), Überlandstrasse 129, \\ CH-8600 Dübendorf, Switzerland;
}

\begin{abstract}
Lensless imaging requires coherent illumination, which is typically available at beamlines. A study of lensless imaging with a table-top X-ray laser is presented, comparing two alternative data-processing techniques, i.e. using the near and the far field data. In order to have a consistent comparison, a Fresnel zone plate was studied as object, because its modulated structure permits to obtain both Fresnel (near field) and Fraunhofer (far field) pattern simultaneously. Results show factor of 3 difference in imaging resolution, and a major limitation given by the degree of the illumination homogeneity.
\end{abstract}

Keywords: Plasma, X-ray Laser, Lensless, Tabletop, Data Science, HIO, FBP, materials characterization

\section{INTRODUCTION}

A number of processes in nature and technology are occurring with characteristic length-scales at the limit of the optical resolution and/or well-above it. From micro/nano-particle assemblies to surface roughness distributions, materials samples may also be polydispersed, which requires a multiscale imaging capability. Furthermore, during operando experiments, the capability for simultaneous imaging below and above the micron threshold is crucial for field surveying and feature zooming of a sample.

Classical light imaging experiments rely on the utilization of optics. ${ }^{1}$ Besides the illumination, the specific geometrical characteristics as well as the finishing quality of the optics do limit the imaging specifications. Indeed, optical resolution is given by the illumination wavelength, the numerical aperture (NA) of light collection (bandwidth), and the finishing quality of the optics. In the case of multi-optical systems the geometry of the optical scheme is the dominating factor, limiting the resolution at large NA imaging, as shown previously in Ruiz-Lopez et al. ${ }^{2}$ while using a self-developed Schwarzschild objective.

Alternatively, electron-beam imaging methods, either in scanning (SEM) or transmission (TEM) microscopy, are extremely powerful to access the low nano-scale in commercially available systems. However, sample preparation procedures, especially needed if the sample is non-conductive, limit the possibility of direct, operando, and high spatial resolution application. Simultaneous imaging at nano and micro scale is not possible with either SEM or TEM, because the pixel size is constant and the chosen field of view determines the ultimate resolution.

Hence, despite the limitations dictated by the optics, light imaging carries significant advantages of directness, full field and multiscale capability. Therefore, several studies have focused on the development of photon-based lensless imaging techniques. Lensless imaging is based on image retrieval from a diffraction pattern, produced under coherent illumination. ${ }^{3}$ The pattern is indeed modulated to the characteristic length-scale of the object, and a phase-retrieval algorithm is necessary to reconstruct the object.

Until now, most of the lensless experiments at short-wavelength have been done at accelerator facilities. In fact, so far, only free-electron laser and synchrotron provided the needed coherence and brightness at short wavelengths ${ }^{4}$ for such photon-hungry experiments. However, recently, few groups have tackled the challenge of lensless imaging with table-top sources. ${ }^{5-7}$ Ideally, highly monochromatic pulses are needed, in order to obtain sharp fringes and permit the object reconstruction. High Harmonic Generation (HHG) has been utilized but its modest brightness and wide bandwidth are major limitations.

Present address for MRL: Deutsches Elektronen-Synchrotron, DESY, Notkestrasse 85, 22607 Hamburg, Germany

International Conference on X-Ray Lasers 2020, edited by Davide Bleiner,

Proc. of SPIE Vol. 11886, 118860P - (C) 2021 SPIE · CCC code:

0277-786X/21/\$21 - doi: $10.1117 / 12.2596041$

Proc. of SPIE Vol. $11886118860 \mathrm{P}-1$ 
On the other hand, the plasma-based amplified spontaneous emission (ASE) X-ray laser, with less than $10^{-4}$ relative bandwidth (which also enhances the peak brigthness) is potentially optimal for table-top coherent diffraction imaging. Therefore, we experimentally investigated lensless imaging in the X-ray using a table-top plasma-laser. Such X-ray laser was shown previously to be fully coherent in time, but only partially (10-20\%) in space. ${ }^{2}$

The paper is organized as follows: Section 2 describes the experimental setup and the two methods used for the reconstruction. Section 3 discussed the image reconstruction results and in Section 4 conclusions are summarized.

\section{THEORY \& METHODS}

Fig. 1 shows the theoretical dependence of the spatial coherence degree versus the beam width. The beam expands as a Gaussian propagation in the horizontal axis. In the near-field (Fresnel) regime the highest spatial coherence degree is obtained, while in the far-field (Fraunhofer) regime, the spatial coherence tends to degrade. The quality of the reconstruction is affected by the degree of spatial coherence. Reconstructed areas with poorer contrast may be due to lower spatial coherence. The transition from Fresnel to Fraunhofer is not merely a function of collection distance but depends on the scattering feature length-scale. In a object with multiscale features, one can have Fresnel and Fraunhofer in concomitance.

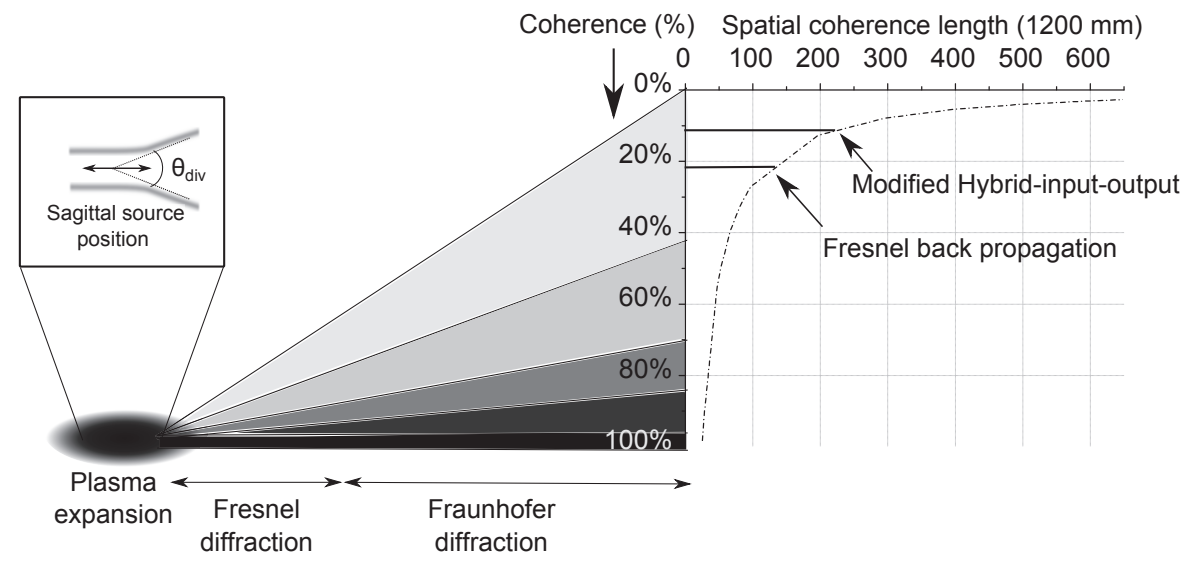

Figure 1. Spatial coherence length at $\mathrm{z}=1.2 \mathrm{~m}$ of propagation. Fresnel back propagation requires higher coherence than the modified hybrid-input-output method, since the former is a convolution of the sample and the source.

As known, the transition between near and far field diffraction is given by the Fresnel number $\left(\mathrm{F}_{N}\right)$ as follows:

$$
F_{N}=\frac{a^{2}}{\lambda \cdot L_{d}}
$$

where $a$ is the sample length-scale, $\lambda$ is the wavelength and $L_{d}$ is the distance between the sample and the detector. ${ }^{8}$ For $\mathrm{F}_{N}>>1$, the diffracted radiation is near-field imaged, or otherwise far-field for $\mathrm{F}_{N}<<1$. Multiscale lensless imaging is important for the investigation of complex samples that are characterized from a large range of roughness scales. An extremely high monochromatic X-ray source is important to obtain rapid and efficient data processing.

The illumination of a Fresnel zone plate with a plasma X-ray laser (XPL) is a template diffraction experiment for this purpose, as it provides simultaneously Fraunhofer diffraction for the outer rings and Fresnel diffraction for the inner ones, while XPL is known to have $\frac{\Delta \lambda}{\lambda} \leq 0.01 \%$. This configuration allows comparing two alternative reconstruction methods, i.e. Hybrid-Input-Output (HIO) and Fresnel Back-Propagation (FBP) methods, with consistent and unique operating conditions (Fig. 1). 
A data-processing method for lensless reconstruction known for its simplicity and noise-resilient performance, is the so-called HIO algorithm. It is a variant of the popular phase-retrieval Gerchberg-Saxton (GS) algorithm. ${ }^{9}$ In GS the information that is used to solve the phase problem is the measured intensity distribution in both the image and object planes, while the two are Fourier-related. The accuracy of the outcome and convergence speed depend on the initialisation phase guess. Algorithms like the modified HIO overcome the problem by using a bias-free approach for the reconstruction of the phase.

On the other hand, FBP is an alternative methodology for objects with larger features at a given imaging plane. The FBP algorithm is based on the wave propagation integral, which in the near field carries a quadratic dependence, ${ }^{10}$ while for the far field the wavefront integral is linearized. Far-field diffraction imaging is often degraded due to inhomogeneous illumination of the diffraction pattern.

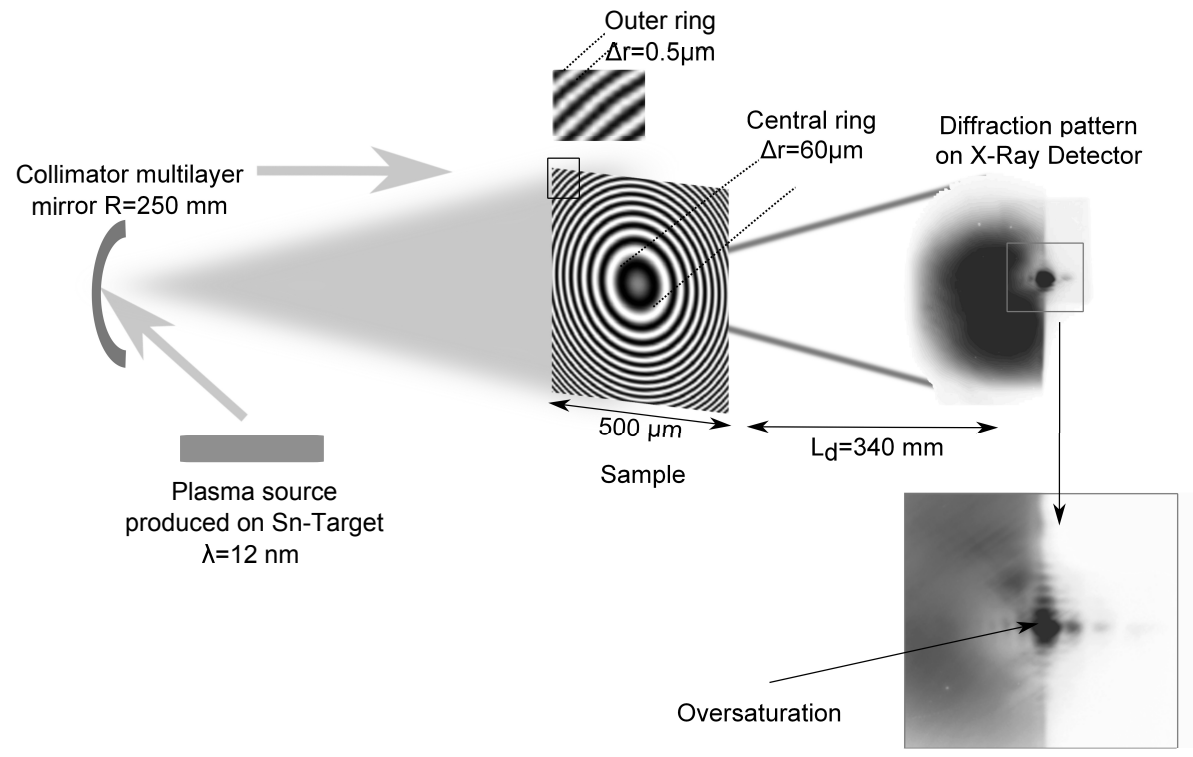

Figure 2. Schematic of the experimental setup. The laser is collimated by using a spherical multilayer mirror to engance the illumination, as explained previously. ${ }^{2}$ The illuminated the sample is a zone plate, and the coherent diffraction pattern is imaged.

\subsection{Experimental Setups}

The experiments were performed at the in-house TW-laser facility (now upgraded as EMPULSE, formerly Beagle). The experimental set-up is illustrated in Fig. 2. The $1054 \mathrm{~nm}$ Nd:glass laser, with two pre-pulses (0.5 and $8 \%$ are the respective amplitudes to the total pump energy) with identical pulse duration as the main pulse (1.2 ps), delivered ca. $3 \mathrm{~J}$ on a tin $(\mathrm{Sn})$ planar target across a 12-mm-long line-focus. A X-ray illumination fluence of ca. $0.03 \mathrm{~J} / \mathrm{cm}^{2}$ at a wavelength of $\lambda=11.97 \mathrm{~nm}$ was utilized. The X-ray pulses are driven to the sample by using a spherical multilayer mirror of $250 \mathrm{~mm}$ radius of curvature. The mirror is, as well, used to collimate the divergence of the beam, ${ }^{2}$ obtaining for this experiment a beam diameter, at the sample, of $0.6 \mathrm{~mm}$.

The illuminated sample was a Fresnel Zone Plate (FZP), fabricated with electron-beam lithography on a $S_{3} N_{4}$ membrane, with a diameter of $0.5 \mathrm{~mm}$ and outer ring size of $0.5 \mu \mathrm{m}$ for a total of 250 rings. Due to the modulated space between the FZP rings, as an object for diffraction experiments, it produced patterns in the near and in the far field simultaneously. The sample was also modelled using a Matlab code and also imaged with a 10X-Leica DM6000M optical microscope.

\subsection{Image-Retrieval Methods}

The HIO algorithm was deployed for the part of diffraction giving a Fraunhofer pattern. Alternatively, for the Fresnel component of the FZP diffraction, a back-propagation method was carried out. 


\subsubsection{Modifield Hybrid-Input-Output Method}

A modified Hybrid-Input-Output (HIO) algorithm was adopted. The pattern imaged experimentally is assumed to be the Fourier Transform of the diffracting object. Hence, the reconstruction procedure relates the collected pattern and the sample object as follows:

$$
\begin{aligned}
\mathfrak{F}\{f(x)\} & =\mathfrak{F}(u)=\mathfrak{F}(u) \cdot(i \cdot \exp (\varphi)) \\
\mathfrak{F}^{-1}\{\mathfrak{F}(u)\} & =f(x)=f(x) \cdot(i \cdot \exp (\phi))
\end{aligned}
$$

The direct and inverse relations are shown, where $\mathfrak{F}(u)$ and $f(\mathrm{x})$ are the function amplitude in spectral and object domain, and $\varphi$ and $\phi$ are the phase in the spectral and the object domain, respectively. The classical GS implementation of this concept is based on four steps, namely:

1. Fourier Transform of a reference model-object,

2. Replacing the modulus with the measured amplitude,

3. Inverse Fourier Transform of the obtained pattern,

4. Replacing the modulus of the resulting computational image with the Fourier Transform amplitude of the experimental object (and initiate a new iteration until convergence).

In the Fienup algorithm ${ }^{11}$ or hybrid-input-output algorithm (HIO), the fourth step is skipped in place of a new iteration directly after the FT step. In each iteration the error reduces up to a point where the matching of the experiment and the model cannot get closer. The residual error was calculated as follows:

$$
\epsilon_{k}^{2}=\int\left(S(u)-M(u)_{k}\right)^{2} d u
$$

where $M(u)_{k}$ is the real part of the retrieval object at the iteration $k$ and $S(u)$ is the modulus of the calculated object, which is used as a guess for the next iteration. A method to relax the error was introduced by Bauschke et al., ${ }^{12}$ for which $\mathrm{S}(\mathrm{u})$ is described as follows:

$$
S^{\prime}(u)=\left(C_{r e l} \cdot m(x)\right)+\left(1-C_{r e l} \cdot S(u)\right)
$$

where $S^{\prime}(u)$ is the new modulus in the spectral domain after introducing the relaxation coefficient $C_{r e l}$. The relaxation factor is included in the algorithm at the place of the step two, introducing only a percentage of the experimental module and complete it with a modulus-unit such $m(x)=1$ in Eq. 4 .

\subsubsection{Fresnel Back-Propagation Method}

A Fresnel back propagation (FBP) algorithm is based on the optical analysis of wave propagation. Fresnel propagation is a quadratic approximation of the Kirchhoff integral, valid in the near-field, where the wavefront curvature is important. ${ }^{13}$ The propagation of a Gaussian beam is based on the expansion of a wavelet function from the object. The Fresnel propagation and diffraction are described at the collection point $\left(E\left(x_{0}, y_{0}\right)\right)$ as follows:

$$
E\left(x_{0}, y_{0}\right)=\frac{e^{i k z}}{i \lambda z} \iint_{-\infty}^{+\infty} O\left(x_{0}, y_{0}\right) e^{\left(\frac{i k}{2 z}\right)\left[\left(x-x_{o}\right)^{2}+\left(y-y_{0}\right)^{2}\right]} d x d y
$$

where $O\left(x_{0}, y_{0}\right)$ defines the dimensions of the sample object and $k$ is the wavenumber of the light propagated along $z$ with wavelength $\lambda$. In the FBP algorithm the phase is assumed to be constant to simplify the reconstruction. The retrieved image was experimentally collected with a magnification of 2.4 -fold. ${ }^{14}$ 


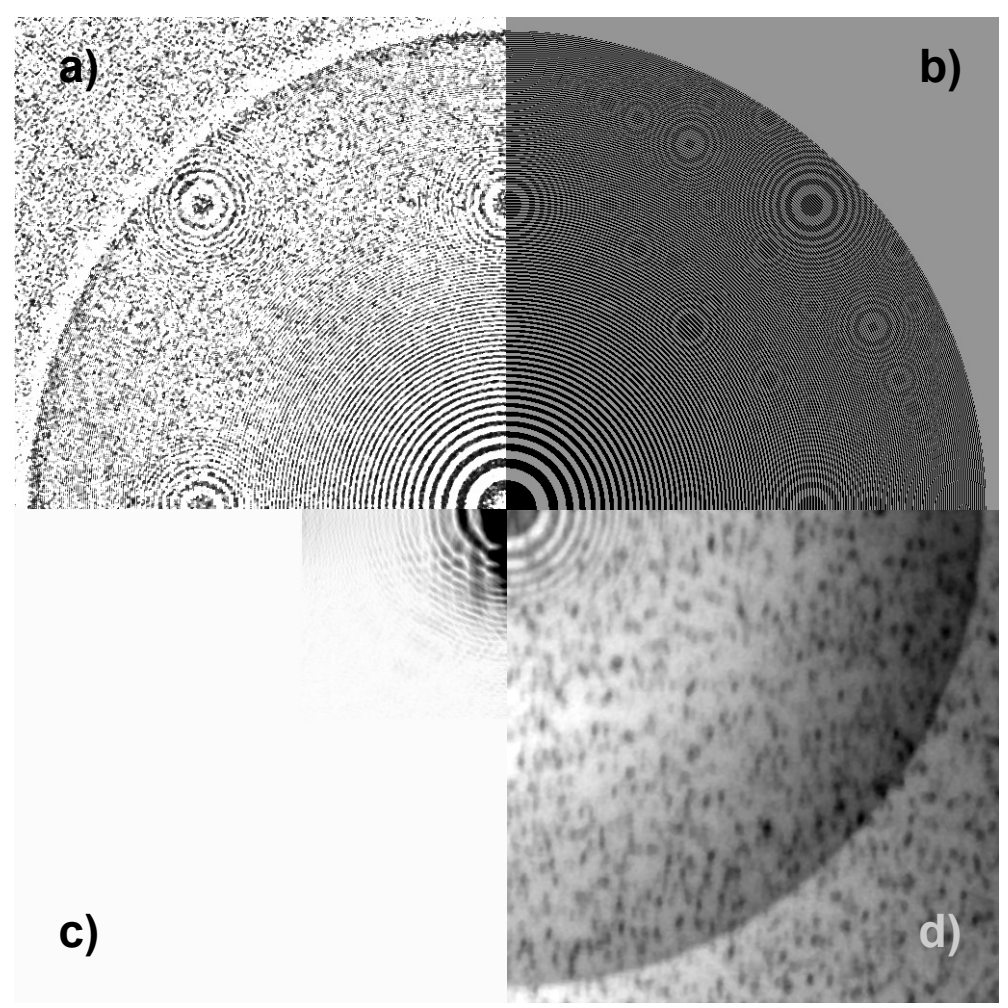

Figure 3. a) Reconstruction image using the modified hybrid input-output algorithm, b) Fresnel Zone Plate estimated using Matlab. The image was used as a model for the hybrid input-output algorithm. c) Reconstruction image using Fresnel Back Propagation. d) Photography of the Zone Plate at the optical microscope.

\section{RESULTS AND DISCUSSION}

Fig. 3 compares the experimental images retrieved with the data processing algorithms HIO (Fig. 3.a) and FBP (Fig. 3.c), with an estimation of the sample shape obtained computationally (Fig. 3.b) and a image at the optical microscope (Fig. 3.b). Fraunhofer diffraction orders are observed at the center. The black semi-circle around is the Fresnel pattern. The Fraunhofer pattern shows over-saturation near the zeroth order. As a consequence there is a lack of information for high frequencies (the outer part of the Fresnel Zone Plate) in the reconstruction, as observed in Fig. 3.a. and b.

The uniformity of the illumination is essential to perform coherent diffractive imaging as well as the brightness of the pulse. In a previous study, we were able to demonstrate that the ASE X-ray laser profile across a plasma gain-medium could be optically controlled to obtain an improved illumination homogeneity. ${ }^{2}$ Still the performance is not satisfactory to obtain high quality images.

Fig. 4 shows the reconstruction of the Fresnel Zone Plate using: a) the HIO algorithm and b) the FBP algorithm. Both retrievals are compared with a estimation of the real Frenel Zone Plate. The HIO reconstruction shows a white central peak due to the over-saturation at the zeroth order in the diffraction pattern. The red line shows the range of the profiles in $\mathrm{c}$ ) and $\mathrm{d}$ ).

The visibility of the pattern is defined by illumination characteristics such as brightness and homogeneity. Due to the high brightness of the X-ray plasma-laser $\left(>10^{26} \mathrm{phs}^{-1} \mathrm{~mm}^{-2} \mathrm{mrad}^{-2} 0.1 \% \mathrm{BW}\right)$ and the collimation (full angle $\left.\sigma_{d i v}=0.5 \mathrm{mrad}\right),{ }^{2}$ the nominal source conditions for lensless imaging are fulfilled. The presence of noise may perturb the convergence of the algorithm. Before the algorithm was applied, the CCD image was background-corrected. The initial value of signal-to-noise ratio was 5.7 and after the correction the signal-to-noise ratio was 9.0.

Fig. 5 shows the experimental RMS error for the HIO algorithm. The initialisation error of $\varepsilon \simeq 0.6$ (RMS) decreases as low as 0.3 if no relaxation coefficient is used. However, by using a relaxation coefficient of $\mathrm{C}_{r e l}=0.5$ 
a)

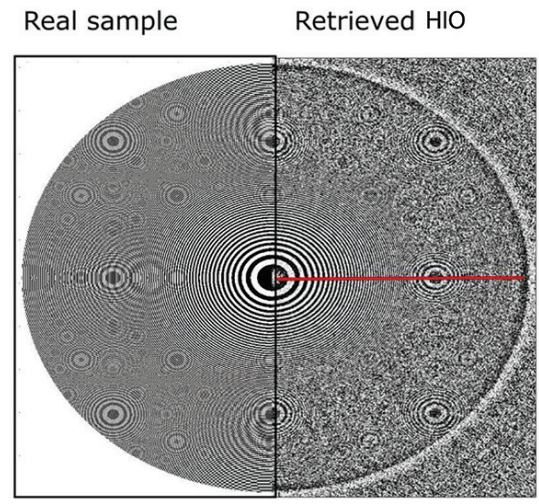

c)

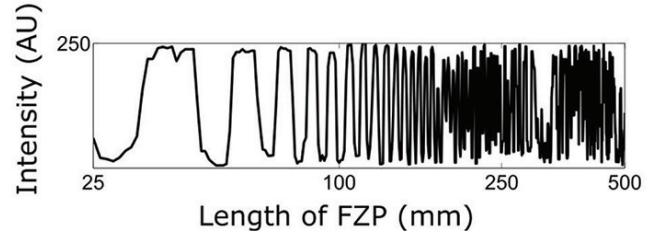

b)

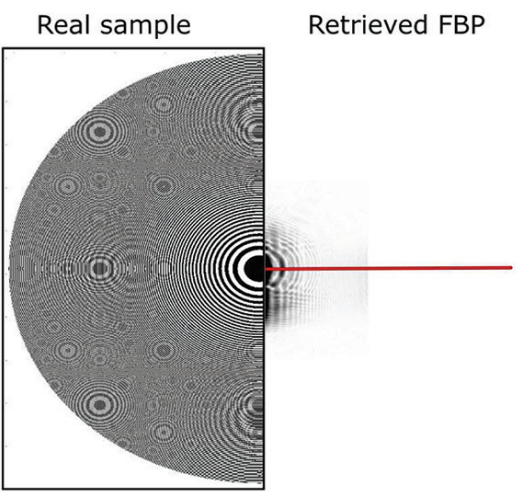

d)

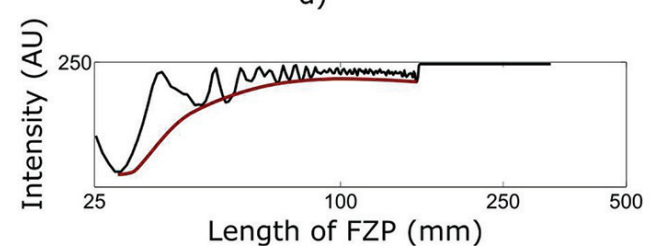

Figure 4. Reconstruction of the sample using a) HIO method (LHS simulated, RHS experimetal retrieval) and b) FBP method (LHS simulated, RHS experimetal retrieval) and radial profiles c) HIO and d) FBP

the RMS error is $\varepsilon \simeq 1.5 \cdot 10^{-3}$ at the iteration $\mathrm{k}=57$. After iteration $\mathrm{k}=57$, the RMS error increases up to $\varepsilon \simeq 0.08$ where the plateau establishes. Two main factors influencing the minimum phase-error reachable were found the following ones: (i) the similitude between the model and the object and (ii) the quality of the acquired diffraction patterns.

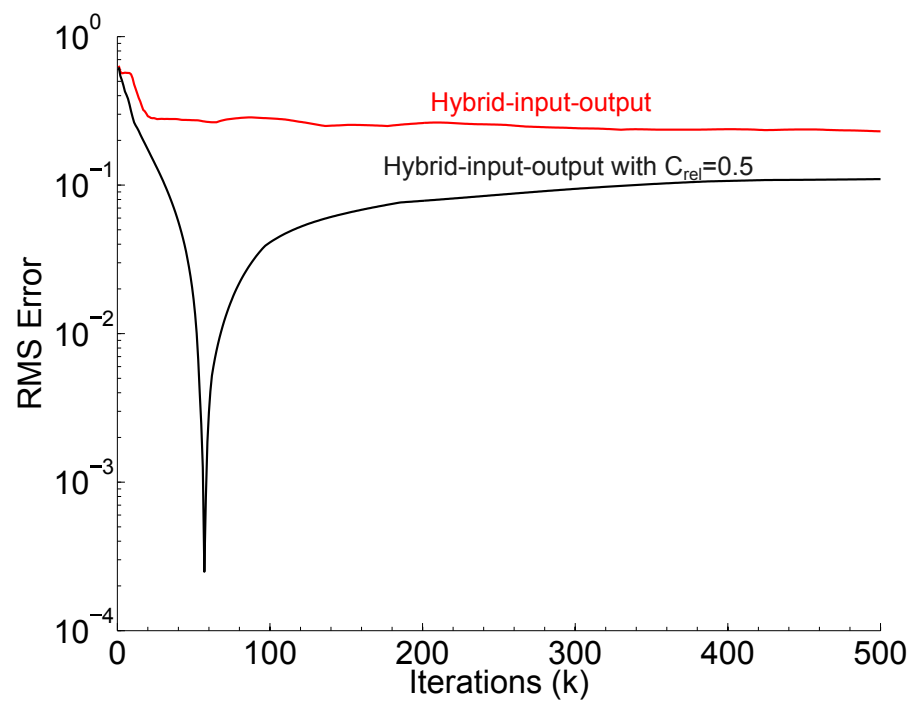

Figure 5. Measured RMS error during the reconstruction as a function of number of iterations. See text for discussion.

The reconstruction carried out with the HIO algorithm allowed a good retrieval up to the $34^{\text {th }}$ ring, with a thickness of $0.96 \mu \mathrm{m}$. The position of ring $34^{\text {th }}$ corresponds to a field of view (FOV) of $200 \mu \mathrm{m}$, with a spatial 
coherence degree $\approx 10 \%$ and intensity contrast $\gamma=0.74$ as shown in Fig. $4 \mathrm{c}$.

The reconstruction of a Fresnel diffraction pattern requires a homogeneous profile of the illumination, in order to analyze independently the image and the sample. A Fresnel pattern is a convolution of the sample and the photon-beam and consecutively contains information about the source. Fig. 4d) shows the profile and the FBP reconstruction. The profile is modeled by a Gaussian function, as follows:

$$
F W H M=\sigma \cdot 2 \cdot \sqrt{2 \cdot \operatorname{Ln} 2}=150 \mu m
$$

which corresponds to a divergence of $0.48 \pm 0.03 \mathrm{mrad}$. This result matches the experimental results obtained in ref. ${ }^{2}$ within the experimental error. Unfortunately, the inhomogeneous illumination still limits the resolution.

The reconstruction carried out by means of the FBP method allowed observing as far as the $18^{\text {th }}$ ring, with a thickness of $2.7 \mu \mathrm{m}$. The position of such ring corresponds to a spatial coherence degree of $22 \%$ and an intensity contrast $\gamma=0.67$.
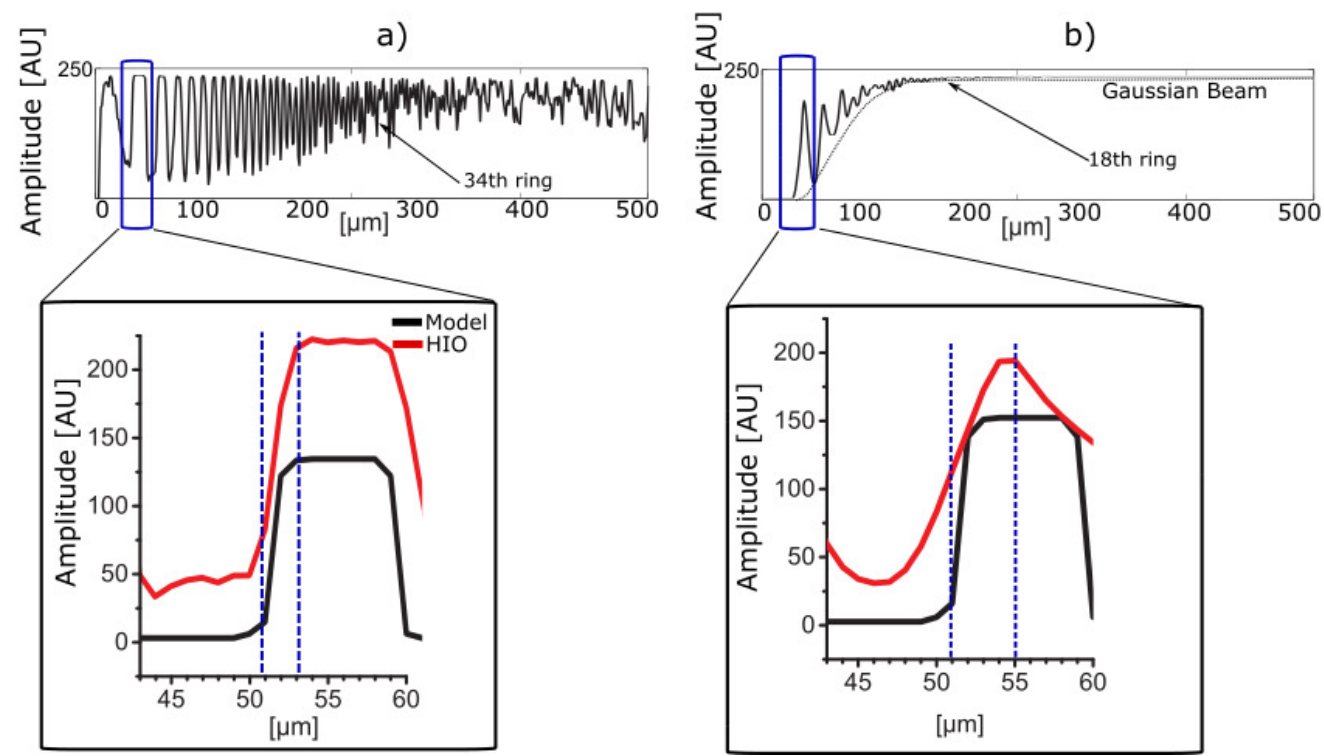

Figure 6. a) Profile of the reconstructed sample using the modified hybrid input-output algorithm. b) Profile of the reconstructed sample using the Fresnel back propagation. The highest intensity (255) corresponds to white and the lowest intensity (0) to black. The box below compared the contrast in the second ring between the reconstruction and the model calculated with Matlab.

Fig. 6 compares the profile of the reference, with the (a) HIO algorithm reconstruction and (b) the FBP algorithm reconstruction. In the vertical axis, highest values of the amplitude correspond to white (255) and lower values correspond to black (0). The central rings of the Fresnel Zone Plate conserve a good contrast. In the FBP method the unknown function is the object, since the observation field is given at the detector with the diffraction pattern. The non-homogeneity and limited coherence, effect of pulse formation across the plasma medium, remain printed in the reconstruction.

\section{CONCLUSIONS}

The use of plasma-driven X-ray lasers for imaging is attractive (see also Sects. 1-4 [15]). Different data processing algorithms were tested. An imaging spatial resolution of $0.9 \mu \mathrm{m}$ was demonstrated with the HIO. Fresnel reconstruction reached a reso-lution of $2.7 \mu \mathrm{m}$, even though the latter did not need information about the phase for the reconstruction a priori. The second method provides also metrology measurements of the beam. Multiscale Fresnel lensless imaging was demonstrated to work across a factor of 3 length-scale span. 


\section{REFERENCES}

1. D. Bleiner, F. Staub, V. Guzenko, Y. Ekinci, and J. E. Balmer, "Evaluation of lab-scale euv microscopy using a table-top laser source," Optics Communications 284(19), pp. 4577-4583, 2011.

2. M. Ruiz-Lopez and D. Bleiner, "Implementing the plasma-lasing potential for tabletop nano-imaging," Applied Physics B 115(3), pp. 311-324, 2014.

3. D. Attwood, Soft x-rays and extreme ultraviolet radiation: principles and applications, Cambridge university press, 1999.

4. J. Miao, RL. Sandberg, and CSong, "Coherent x-ray diffraction i maging," Selected Bpics in Quantum Electronics, IEEE Journal of 18(1), pp. 399-410, 2012.

5. R. L. Sandberg, CSong, P. W. Wachulak, DA. Rymondson, A. Paul, B Amirbekian, E Lee, A. E Sakdinawat, L.O. Gan, M. CMarconi, et al., "High numerical aperture tabletop soft x-ray diffraction microscopy with 70-nm resolution," Proceedings of the National Academy of Sciences 105(1), pp. 24-27, 2008.

6. S. Witte, VT. Tenner, DW. Noom, and K. S. Fikema, "Lensless diffractive i maging ith ultra-broadband tabletop sources: f rom i nfrared to extreme-ultraviolet velengths," Light: Science EA pplications 3(3), p. e163, 2014.

7. D. F. Gardner, M. Tanksalvala, E. R. Shanblatt, X. Zhang, B. R. Galloway, C. L. Porter, R. Karl Jr, C. Bevis, D. E. Adams, H. C. Kapteyn, et al., "Subwavelength coherent imaging of periodic samples using a $13.5 \mathrm{~nm}$ tabletop high-harmonic light source," Nature Photonics 11(4), p. 259, 2017.

8. L. Sveda and L. Pina, "Simulations of lensless imaging in water window," in Optics and Measurement International Conference 2016, 10151, p. 101510E, International Society for Optics and Photonics, 2016.

9. R. W. Grchberg, "A practical algorithm $\mathrm{f}$ or the determination of phase $\mathrm{f}$ rom i mage and diffraction plane pictures," Optik 35, p. 237, 1972.

10. D. Schreier, Synthetische holografie, Fachbuchverl. Leipzig, 1984.

11. J. R. Fienup, "Phase retrieval algorithms: a comparison," Applied optics 21(15), pp. 2758-2769, 1982.

12. H. H. Buschke, P. L. Gmbettes, and DRLuke, "Hybrid projection-reflection rthod f or phase retrieval," JOSA A 20(6), pp. 1025-1034, 2003.

13. P. Enders, "Huygens' principle and the modelling of propagation," European Journal of Physics 17(4), p. 226, 1996.

14. M. Ruiz-Lopez, A. Faenov, T. Pikuz, N. Ozaki, A. Mitrofanov, B. Albertazzi, N. Hartley, T. Matsuoka, R. Ochante, YTange, et al., "Coherent x-ray beam ætrology using $2 \mathrm{~d}$ high-resolution $\mathrm{f}$ resnel-diffraction analysis," Journal of synchrotron r adiation 24(1), pp. 196-204, 2017

15. D. Bleiner, The Science and Technology of X-ray Lasers: A 2020 Update Proc. SPIE 11886, 1188602 (2021) 